\title{
From Order to Chaos
}




\section{WORLD SCIENTIFIC SERIES ON NONLINEAR SCIENCE}

Editor: Leon O. Chua

University of California, Berkeley

\section{Series A. MONOGRAPHS AND TREATISES}

Volume 1: From Order to Chaos

L. P. Kadanoff

Volume 2: Ideal Turbulence

A. N. Sharkovsky, E. Yu. Romanenko, and M. B. Vereikina

Volume 3: Dynamics of Piecewise-Monotone Maps

A. N. Sharkovsky, S. F. Kolyada, and A. G. Sivak

Volume 4: Qualitative Tools of Nonlinear Dynamics

Part 1: Local and Global Bifurcations

L. O. Chua, A. L. Shil'nikov, L. P. Shil'nikov, and D. V. Turaev

Volume 5: Qualitative Tools of Nonlinear Dynamics

Part 2: Homoclinic Chaos

L. O. Chua, A. L. Shil'nikov, L. P. Shil'nikov, and D. V. Turaev

Volume 6: Stability, Structures and Chaos in Nonlinear Synchronization Networks

V. S. Afraimovic, V. I. Nekorkin, G. V. Osipov, and V. D. Shalfeev

Edited by Academicians A. V. Gaponov-Grekhov and M. I. Rabinvich

Volume 7: Smooth Invariant Manifolds and Normal Forms

1. U. Bronstein and A. Ya. Kopanskii

Volume 8: Dynamical Chaos: Models, Experiments, and Applications V. S. Anishchenko

Volume 9: Frequency Domain Methods for Nonlinear Analysis: Theory and Applications G. A. Leonov, D. V. Ponomarenko, and V. B. Smirnova

Volume 10: Critical Phenomena and Roads to Chaos in Multiparameter and Multidimensional Nonlinear Systems

A. P. Kuznetsov, S. P. Kuznetsov, and I. R. Sataev

Volume 11: Nonlinear Dynamics of Interacting Populations

A. D. Bazykin

Series B. SPECIAL THEME ISSUES AND PROCEEDINGS

Volume 1: Chua's Circuit: A Paradigm for Chaos

R. N. Madan

Volume 2: Complexity and Chaos

N. B. Abraham, A. M. Albano, A. Passamante, P. E. Rapp, and R. Gilmore

Volume 3: New Trends in Pattern Formation in Active Nonlinear Media

V. Perez-Villar, V. Perez-Munuzuri, C. Perez Garcia, and V. I. Krinsky 


\section{FROM ORDER TO CHADS \\ Essalys: Critical, Chaotic and Otherwise}

\section{Leo P. Kadanoff \\ The Research Institutes The University of Chicago \\ USA}




\title{
Published by
}

World Scientific Publishing Co. Ple. Ltd.

P O Box 128, Farrer Road, Singapore 9128

USA office: Suite IB, 1060 Main Street, River Edge, NJ 07661

UK office: 73 Lynton Mead, Totteridge, London N20 8DH

The author and publisher would like to thank the following publishers of the various journals and books for their assistance and permission to include the selected reprints found in this volume:

Academic Press

American Association of Physics Teachers

American Institute of Physics

American Physical Society

Elsevier Science Publishers

Encyclopaedia Britannice, Inc.

European Physical Society

Gordon and Breach Scientific Publishers Inc.

IEEE

La Recherche

Longman Group, UK

National Academy of Sciences

Royal Swedish Academy of Sciences

Society for Computer Simulation

Springer Verlag

\section{FROM ORDER TO CHAOS}

Copyright @ 1993 by World Scientific Publishing Co. Pte. Lid.

All rights resenved. This book, or parts thereof, may not be reproduced in any form or by any means, electronic or mechanical, including photocopying, recording or any information storage and retrieval system now known or to be invented, without written permission from the Publisher.

For photocopying of material in this volume, please pay a copying fee through the Copyright Clearance Center, Inc., 27 Congress Street, Salem, MA 01970, USA.

\author{
ISBN 981-02-1197-X \\ 981-02-1198-8 (pbk)
}

Printed in Singapore. 


\section{Acknowledgements}

This book is an outcome of the exchanges of information with my professors, coworkers, and students. They have all been my teachers. 
This page is intentionally left blank 


\section{CONTENTS}

List of Publications $\quad$ xi

General Introduction: The Worlds of Science 1

Section A. Fundamental Issues in Hydrodynamics, Condensed Matter and Field Theory

$\begin{array}{ll}\text { From Level to Level } & 7\end{array}$

1. On Two Levels [114] 11

2. Hydrodynamic Equations and Correlation Functions [11] 13 (with P. C. Martin)

3. The Electron-Phonon Interaction in Normal and Superconducting Metals [17] 64

4. Wave Function Fluctuations in Finite Superconductors [29] 100

5. The Application of Renormalization Group Techniques to Quarks and Strings [58]

6. Disorder Variables and Para-Fermions in Two-Dimensional Statistical Mechanics [74] (with E. Fradkin)

7. Computational Physics: Pluses and Minuses [115] 149

8. Cathedrals and Other Edifices [117] 151

9. From Neutrinos to Quasiparticles [124] 153

\section{Section B. Scaling and Phase Transitions}

\section{On the Joys of Creation}

1. Scaling Laws for Ising Models Near $T_{\mathrm{c}}[22]$

2. Static Phenomena Near Critical Points: Theory and Experiment [26] 175 (with W. Götze, D. Hamblen, R. Hecht, E. A. S. Lewis, V. V. Palciauskas, M. Rayl, J. Swift, D. Aspnes, and J. W. Kane)

3. Transport Coefficients Near Critical Points [30]

4. The Droplet Model and Scaling [38]

5. Critical Behavior. Universality and Scaling [39]

6. Scaling, Universality and Operator Algebras [54] 240

7. Teaching the Renormalization Group [62] 274 (with H. J. Maris)

8. Scaling and Universality in Statistical Physics [135] 
Section C. Simulations, Urban Studies, and Social Systems

Models and Arguments

1. Computer Display and Analysis of Urban Information

Through Time and Space [37]

301

(with J. R. Voss and W. J. Bouknight)

2. From Simulation Model to Public Policy: An Examination of Forrester's "Urban Dynamics" [42]

3. Public Policy Conclusions from Urban Growth Models [46] (with $H$. Weinblatt)

4. A Simulation Model of Urban Labor Markets and Development Policy [51]

(with B. Harrison and B. Chinitz)

5. The Big, the Bad and the Beautiful [127]

6. Hard Times [152]

\section{Section D. Turbulence and Chaos}

Questions without Answers

1. Roads to Chaos [93] 391

2. Chaos: A View of Complexity in the Physical Sciences [113] 399

3. From Periodic Motion to Unbounded Chaos: Investigations of the Simple Pendulum [99]

4. Escape from Strange Repellers [96] (with C. Tang)

5. Global Universality at the Onset of Chaos: Results of a Forced Rayleigh-Benard Experiment [106]

(with M. H. Jensen, A. Libchaber, I. Procaccia and J. Stavans)

6. Fractal Measures and Their Singularities: The Characterization of Strange Sets [109]

(with T. C. Halsey, M. H. Jensen, I. Procaccia, and B. I. Shraiman)

7. Fractals: Where's the Physics [110]

8. Scaling and Multiscaling (Fractals and Multifractals) [141]

9. Complex Analytic Methods for Viscous Flows in Two Dimensions [107] 484 (with D. Bensimon, S. Liang, B. I. Shraiman, and C. Tang)

10. On Complexity [119]

11. Interactive Computation for Undergraduates [131]

12. Scaling and Structures in the Hard Turbulence Region of Rayleigh Benard Convection [134] 
13. Turbulence dans une Boîte [147] (with A. Libchaber, E. Moses and G. Zocchi)

14. Complex Structures from Simple Systems [149]

15. Bubble, Bubble, Boil and Trouble [151]

539 (with D. H. Rothman)

Numbers in brackets refer to the numbers in the list of publications on pp. xi-xvii. 
This page is intentionally left blank 


\section{Publications of Leo Kadanoff}

1. (with P. C. Martin) Knight shift in superconductors, Phys. Rev. Letts. 3, 322 (1959).

2. (with P. C. Martin) Transport properties of superconductors, Bull. Am. Phys. Soc. 5, 13 (1960).

3. Radiative transport within an ablating body, Trans. ASME: Series C 3, 215 (1961).

4. (with V. Ambegaokar) Electromagnetic properties of superconductors, Nuovo Cimento 22, 914 (1961).

5. (with G. Baym) Conservation laws and correlation functions, Phys. Rev. 124, 237 (1961).

6. (with P. C. Martin) Theory of many-particle systems II: Superconductivity, Phys. Rev. 124, 670 (1961).

7. (with G. Baym) Quantum Statistical Mechanics (W. A. Benjamin, New York, 1962), p. 203. Also translated into Russian, Japanese, and Chinese.

8. (with H. Hidalgo) Comparison between theory and flight ablation data, Amer. Inst. of Acronautics and Astronautics J. 1, 41 (1963).

9. Boltzmann equation for polarons, Phys. Rev. 130, 1364 (1963).

10. (with D. Markowitz) Effect of impurities upon critical temperature of anisotropic superconductors, Phys. Rev. 131, 563 (1963).

11. (with P. C. Martin) Hydrodynamic equations and correlation functions, Ann. Phys. 24, 419 (1963).

12. Failure of the electronic quasiparticle picture for nuclear spin relaxation in metals, Phys. Rev. 132, 2073 (1963).

13. (with D. C. Langreth) Perturbation theoretic calculation of polaron mobility, Phys. Rev. 133, A 1070 (1964).

14. (with R. E. Prange) Transport theory for electron-phonon interactions in metals, Phys. Rev. 134, A566 (1964).

15. (with M. Revzen) Green's function formulation of the Feynman model of the polaron, Nuovo Cimento 33, 397 (1964).

16. (with I. I. Falko) Ultrasonic attenuation in superconductors containing magnetic impurities, Phys. Rev. 136, A 1170 (1964).

17. The electron-phonon interaction in normal and superconducting metals, Lectures on the Many-Body Problem, Vol. 2 (Academic Press, New York and London, 1964), p. 787.

18. On the problem of ultrasonic attenuation in superconductors containing magnetic impurities, Proc. 9th Int. Conf. Low Temp. Phys., Columbus, 1964 (Plenum Press, New York, 1965), Part A, pp. 378-80.

19. (with J. W. Kane) Green's functions and superfluid hydrodynamics, J. Math. Phys. 6, $1902(1965)$.

20. (with A. B. Pippard) Ultrasonic attenuation in superconductors, Proc. Roy. Soc. A 292, 299 (1966).

21. Scaling laws for ising models near critical points, Proc. 1966 Midwest Conf. on Theor. Phys. (Bloomington, Ind.).

22. Scaling laws for ising models near Tc, Physics 2, 263 (1966).

23. Spin-spin correlation in the two-dimensional ising model, Nuovo Cimento 44, 276 (1966).

24. Basic Principles of Physics: Electricity, Magnetism, and Heat (W. A. Benjamin, New York, 1967).

25. (with J. W. Kane) Long range order in superfluid Helium, Phys. Rev. 155, 80 (1967).

26. (with W. Götze, D. Hamblen, R. Hecht, E. A. S. Lewis, V. V. Palciauskas, M. Rayl, J. Swift, D. Aspnes, and J. W. Kane) Static phenomen a near critical points: Theory and experiment, Rev. Mod Phys. 39, 395 (1967). 
27. (with J. Swift) Transport coefficients near the critical point: $A$ master-equation approach, Phys. Rev. 165, 310 (1968).

28. (with J. Swift) Transport coefficients near the liquid-gas critical point, Phys. Rev. 166, 89 (1968).

29. Wave function fluctuations in finite superconductors, Comments on Solid State Phys. 1, No. 1 (1968).

30. Transport coefficients near critical points, Comments on Solid State Phys. 1, 5 (1968).

31. (with G. Laramore) Anomalous electrical conductivity above the superconducting transition, Phys. Rev. 175, 579 (1968).

32. (with J. Swift) Transport coefficients near the L transition of helium, Ann. Phys. 50, 312 (1968).

33. Operator algebra and the determination of critical indices, Phys. Rev. Letts. 23, 1430 (1969).

34. Correlations along a line in the two-dimensional ising model, Phys. Rev. 188, 859 (1969).

35. (with J. R. Voss and W. J. Bouknight) A city grows before your eyes, Computer Decisions (Dec., 1969).

36. (with G. E. Laramore) Anomalous ultrasonic attenuation above the magnetic critical point, Phys. Rev. 187, 619 (1969).

37. (with J. R. Voss and W. J. Bouknight) Computer display and analysis through time and space, Technological Forecasting and Social Change 2, 77 (1970).

38. The droplet model and scaling, Critical Phenomena, Proc. Int. School of Physics, "Enrico Fermi", Course LI, ed. M. S. Green (Academic Press, New York, 1971), p. 118.

39. Critical behavior universality and scaling, Critical Phenomena, Proc. Int. School of Physics, "Enrico Fermi", Course LI, ed. M. S. Green, (Academic Press, New York, 1971), p. 100.

40. Studying and displaying urban growth patterns, Aerospace and Electronic Systems 7, No. 3 (1971).

41. (with H. Ceva) Determination of an operator algebra for the two-dimensional ising model, Phys. Rev. B3, 3918 (1971).

42. From simulation model to public policy: An examination of Forrester's urban dynamics, Simulation 16, 261 (1971). Reprinted in Best Computer Papers of 1971, ed. O. Petrocelli (Anerbach, New York, 1971).

43. (with F. J. Wegner) Some critical properties of the eight-vertex model, Phys. Rev. B4, 2909 (1971).

44. A Modified Forrester Model of the United States as a Group of Metropolitan Areas, Urban Dynamics: Extensions and Reflections (San Francisco Press, 1972).

45. (with H. Weinblatt) Public Policy Conclusions from Urban Growth Models Urban Dynamics: Extensions and Reflections (San Francisco Press, 1972).

46. (with $\mathrm{H}$. Weinblatt) Public policy conclusions from urban growth models, IEEE Trans. on Systems, Man, and Cybernetics, SMC-2, 159 (1972).

47. (with M. K. Grover and F. J. Wegner) Critical exponents for the Heisenberg model, Phys. Rev. B6, 311 (1972).

48. (with B. Chinitz, G. Crampton, S. Jacobs, J. Tucker, and H. Weinblatt) The Brown University National Metropolitan Models, Socio-Economic Systems and Principles, ed. W. Vogt, et al. (Univ. Pittsburgh, Pennsylvania, 1973). Also published in Synergetics, ed. A. Haken (B. G. Taubner, Stuttgart, 1973).

49. Renormalization equations: conceptual basis and a simple example, Renormalization Group in Critical Phenomena and Quantum Field Theory, ed. J. D. Gunton and M. S. Green (Temple Univ. Press, Philadelphia, 1973), p. 21. 
50. Renormalization group techniques on a lattice, Cooperative Phenomena, ed. H. Haken (North Holland, 1974), p. 139.

51. (with B. Harrison and B. Chinitz) A Simulation model of urban labor markets and development policy, Urban Development Models, ed. R. Baxter, et al. (The Construction Press, 1975).

52. (with A. Houghton) Numerical evaluations of the critical properties of the twodimensional ising model, Phys. Rev. B11, 377 (1975).

53. Variational principles and approximate renormalization group calculations, Phys. Rev. Letts. 16, 1005 (1975).

54. Scaling, universality, and operator algebras, Phase Transitions and Critical Phenomena, ed. C. Domb and M. S. Green, Vol. 5A (Academic Press, New York, 1976), p. 1 .

55. From simulation model to public policy, The American Scientist 60, 74 (1972).

56. (with A. Houghton and M. C. Yalabik) Variational approximations for renormalization group transformations, J. Stat. Phys. 14, 171 (1976). Also Published in Proc. IUPAP Statistical Mechanics Conference (Hungarian Academy of Sciences, 1976).

57. Notes on Migdal's recursion formulas, Ann. Phys. 100, 359 (1976).

58. The application of renormalization group techniques to quarks and strings, Rev. Mod. Phys. 49, 267 (1977). Also published in Lecture Notes in Physics 54 (Springer-Verlag, 1976), p. 276.

59. (with J. Jose, S. Kirkpatrick, and D. Nelson) Renormalization, vortices, and symmetry breaking interactions in the $\mathrm{d}=2$ planar model, Phys. Rev. B16, 1217 (1977).

60. Connections between the critical behavior of the planar model and that of the eightvertex model, Phys. Rev. Letts. 39, 903 (1977).

61. A Model for Interacting Quarks and Strings, Proc. IUPAP Statistical Mechanics Conference, Haifa, 1977.

62. (with H. Maris) Teaching the renormalization group, Am. J. Phys. 46, 652 (1978).

63. Lattice Coulomb gas representation of two-dimensional problems, J. Phys. A11, 1399 (1978).

64. (with Scott J. Shenker and A. Pruisken) A variational real space renormalization group transformation based on the cumulant expansion, J. Phys. A12, 91 (1979).

65. Multicritical behavior at the Kosterlitz-Thouless critical point, Ann. Phys. 120, 39 (1979).

66. (with R. Ditzian) High temperature series expansion methods for ising systems with quenched impurities, Phys. Rev. 19, 4531 (1979).

67. (with A. Brown) Correlation functions on the critical lines of the Baxter and AshkinTeller models, Ann. Phys. 121, 319 (1979).

68. (with R. Ditzian) Series studies of the four state Potts model, J. Phys. A12, L229 (1979).

69. (with M. Kohmoto) SMJ's analysis of ising model correlation functions, Ann. Phys. A $126,371(1980)$.

70. (with R. Ditzian, J. R. Banavar, and G. S. Grest) Phase diagram for the Ashkin-Teller model in three dimensions, Phys. Rev. 22, 2542 (1980).

71. Singularities near the bifurcation point of the Ashkin-Teller model, Phys. Rev. B2, 1405 (1980).

72. (with A. M. M. Pruisken) Marginality, universality and expansion techniques for critical lines in two dimension, Phys. Rev. B22, 5154 (1980).

73. Lower bound RSRG approximation for a large h system, J. Phys. A13, 3339 (1980).

74. (with E. Fradkin) Disorder variables and para-fermions in two-dimensional statistical mechanics, Nucl. Phys. B170, 1 (1980).

75. (with A. N. Berker) Ground state entropy and algebraic order at low temperatures, J. Phys. A13, L259 (1980). 
76. (with A. Zisook) Planar model correlation functions, J. Phys. A13, L379 (1980).

77. (with A. Zisook) Correlation functions on the critical line of the two-dimensional planar model: Logarithms and correlations to scaling, Nucl. Phys. B180, 61 (1981).

78. (with M. Kohmoto) Quantum mechanical ground states, non-linear Schrodinger equations, and linked cluster expansions, J. Phys. A 14, 1291 (1981).

79. (with Scott J. Shenker) Band to band hopping in one-dimensional maps, J. Phys. A14, L23 (1981).

80. (with $M$. Kohmoto and $M$. den Nijs) Hamiltonian studies of the $d=2$ Ashkin-Teller model, Phys. Rev. B24, 5229 (1981).

81. (with M. Kohmoto) Disorder variables for a non-abelian symmetry group, Nucl. Phys. B180, 671 (1981).

82. Many point correlation functions in a modified ising model, Phys. Rev. B24, 5382 (1981).

83. (with Scott J. Shenker) Critical behavior of a KAM surface: I. empirical results, $J$. Stat. Phys. 27, 631 (1982).

84. Scaling for a critical KAM trajectory, Phys. Rev. Letts. 47, 1641 (1981).

85. Critical behavior of a KAM surface: II. renormalization approach, Melting, Localization, and Chaos, ed. R. K. Kalia and P. Vashista (North Holland, New York, 1982).

86. (with $\mathrm{M}$. Widom) Renormalization group analysis of bifurcations in area preserving maps, Physica 5D, 287 (1982).

87. (with M. Feigenbaum and Scott J. Shenker) Quasiperiodicity in dissipative systems: A renormalization group analysis, Physica 5D, 370 (1982).

88. (with S. Howes and $\mathrm{M}$. den Nijs) Quantum model for commensurate-incommensurate transitions, Nucl. Phys. B 215,169 (1983).

89. (with E. Domany, S. Alexander, and D. Bensimon) Solutions to the Schrodinger equation on some fractal lattices, Phys. Rev. B28, 3110 (1983).

90. Supercritical behavior of an ordered trajectory, J. Stat. Phys. 31, 1 (1983).

91. (with M. Kohmoto and C. Tang) Localization problem in one dimension: mapping and escape, Phys. Rev. Letts. 50, 1870 (1983).

92. (with D. Bensimon and Scott J. Shenker) Strange objects in the complex plane, M. Widom, J. Stat. Phys. 32, 443 (1983).

93. Roads to Chaos, Phys. Today 30, 46 (1983).

94. Analysis of cycles for a volume preserving map, unpublished.

95. (with D. Bensimon) Extended chaos and disappearance of KAM trajectories, Physica 13D, 82 (1984).

96. (with C. Tang) Escape from strange repellers, Proc. Nat. Acad. Sci. 81, 1276 (1984).

97. Applications of scaling ideas to dynamics, Proc. Erice Summer School on Dynamics, (Springer-Verlag, 1984).

98. (with D. Bensimon and B. Shraiman) Mean-Field Theories for a Ballistic Model of Aggregation, Proc. Kinetics, Aggregation and Gellation Conf., Athens, Georgia, 1984.

99. From periodic motion to unbounded chaos: Investigations of the simple pendulum, Physica Scripta T9, 5 (1985).

100. (with J. R. Banavar and A. A. M. Pruisken) Energy spectrum for a fractal lattice in a magnetic field, Phys. Rev. B31, 1388 (1985).

101. Simulating hydrodynamics: A pedestrian model. J. Stat. Phys. 39, 267 (1985).

102. (with S. Liang) Scaling in a ballistic aggregation model, Phys. Rev. A31, 2628 (1985).

103. (with P. Cvitanovic, M. H. Jensen, and I. Procaccia) Renormalization, unstable manifolds and the fractal structure of mode locking, Phys. Rev. Letts. 55, 343 (1985).

104. Fractal singularities in a measure and how to measure singularities on fractal, Proc. Cargese Summer School, Cargese School, 1985. 
105. (with P. Cvitanovic, M. H. Jensen, and I. Procaccia) Circle maps in the complex plane, Proc. 6th Int. Symposium on 'Fractals in Physics', eds. L. Pietronero and E. Tosatti, (North Holland, 1985).

106. (with M. H. Jensen, A. Libchaber, I. Procaccia, and J. Stavans) Global universality at the onset of chaos: results of a forced Rayleigh-Benard experiment, Phys. Rev. Letts. 55, 2798 (1985).

107. (with D. Bensimon, S. Liang, B. I. Shraiman, and (.. Tang) Complex analy tic methods for viscous flows in two dimensions, Proc. Mexican Summer School, 1985. Also published in Directions in Condensed Matter Physics, ed. G. Grinstein and G. Mazenko (World Scientific, 1986), p. 51.

108. (with D. Bensimon, T. C. Halsey, M. H. Jensen, A. Libchaber, B. I. Shraiman, and J. Siavans) More on microcanonical paradigms, Suppl. to J. Irrep. Events A17, 1597 (1985).

109. (with T. Halsey, M. Jensen, I. Procaccia and B. I. Shraiman) Fractal measures and their singularities: the characterization of strange sets, Phys. Rev. A33, 1141 (1986).

110. Fractals: Where's the Physics, Phys. Today 39, 6 (1986).

111. (with D. Bensimon, S. Liang, B. I. Shraiman, and C. Tang) Viscous flows in two dimensions, Rev. Mex. Fisica 32, S101 (1986).

112. (with D. Bensimon and M. H. Jensen) Renormalization-group analysis of the global structure of the period-doubling attractor, Phys. Rev. A33, 3622 (1986).

113. Chaos: A view of complexity in the physical sciences, The Great Ideas Today (Encyclopedia Brittanica, Inc., Chicago, 1986), p. 86.

114. On two levels, Phys. Today 39, 7 (1986).

115. Computational physics: pluses and minuses, Phys. Today 39, 7 (1986).

116. Saffman-Taylor bubble problem, Unpublished.

117. Cathedrals and other edifices, Phys. Today 39, (1986).

118. Renormalization group analysis of the global properties of a strange attractor, J. Stat. Phys. 43, 395 (1986).

119. On complexity, Phys. Today (March 1987).

120. Dimensional calculations for Julia sets, Proc. European Condensed Matter Physics Congress, April, 1987. Published in Physica Scripta T19, 19-22.

121. (with D. Bensimon) The Breakdown of KAM trajectories, Chaotic Phenomena in Astrophysics, Annals of the New York Academy of Sciences, Vol. 497, pp. 110-16 (1987).

122. (with M. H. Jensen and I. Procaccia) Scaling structure and thermodynamics of strange sets, Phys. Rev. A36, 1409 (August 1987).

123. (with G. Zocchi, B. Shaw, and A. Libchaber) Finger narrowing under local perturbations in the Saftman-Taylor problem, Phys. Rev. A 36, 1894 (August 1987).

124. From neutrinos to quasiparticles. Phys. Today, 7 (August, 1987).

125. (with G. McNamara and G. Zanetti) A Poiseuille Viscometer for lattice gas automata, Complex Systems 1, 791-803 (1987).

126. (with M. Feingold and O. Piro) A way to connect fluid dynamics to dynamical systems: passive scalars, Fractal Aspects of Materials: Disordered Systems, eds. A.J. Hurd, D.A. Weitz and B.B. Mandelbrot (Materials Research Society, Pittsburgh, 1987), p. 203. Diffusion of passive scalars, Fluid Flows: Maps in Three Dimensions. Also appears in Universalities in Condensed Matter, eds. R. Jullian, L. Peliti, R. Rammal and N. Boccara, Springer Proc. Phys. (Springer, Berlin, 1988). 'Transport of passive scalars: KAM surfaces and diffusion in three-dimensional Liouvillean maps, Instabilities and Nonequilibrium Structures, eds. E. Tirapequi and D. Villarroel (D. Reidel, Dordrecht, 1989), p. 37.

127. The big, the bad and the beautiful, Phys. Today 9 (February 1988). 
128. (with G. McNamara and G. Zanetti) From automata to fluid flow: comparisons of simulation and theory, Phys. Rev. A40, 4527 (October 1989).

129. (with $M$. Feingold and $O$. Piro) Passive scalars, 3D volume preserving maps and chaos, J. Stat. Phys. 50, 529 (1988).

130. (with B. Castaing, G. Gunaratne, F. Heslot, A. Libchaber, S. Thomae, Xiao-zhong Wu, S. Zaleski, and G. Zanetti) Scaling of hard thermal turbulence in Rayleigh Benard convection, J. Fluid Mechanics 209, 1 (1989).

131. Interactive computation for undergraduates, Phys. Today 41, 9-11 (December 1988).

132. (with S. Nagel, L. Wu, and S-M. Zhou) Scaling and universality in avalanches, Phys. Rev. A39, 6524 (1989).

133. Fractals and multifractals in avalanche models, Physica D38, 213-214 (1989).

134. Scaling and structures in the hard turbulence region of Rayleigh Benard convection, Proc. Newport Conference on Turbulence (1989), Proc. Europhysics Conference on Turbulence, Moscow (1989).

135. Scaling and universality in statistical physics, Physica A 163, 1-14 (1990).

136. (with W.-S. Dai and S.-M. Zhou) Singularities in complex interface dynamics, Proc. Cargese Conference on Nonlinear Flow Problems (1990).

137. (with C. Amick, S.C.E. Ching, and V. Rom-Kedar) Beyond all orders: singular perturbations in a mapping, J. Nonlinear Science 2, 9-68 (1992).

138. (with X.-Z. Wu, A. Libchaber, and M. Sano) Frequency power spectrum of temperature fluctuations in free convection, Phys. Rev. Letts. 64, 2140-2144 (1990).

139. (with Peter Constantin) Singularities in complex interfaces, Phil. Trans. Roy. Soc London A 333, 379-389 (1990).

140. Exact solutions for the Saffman-Taylor problem with surface tension, Phys. Rev. Letts. 65, 2986-2988 (1990).

141. Scaling and multiscaling (fractals and multifractals), Prac. Second Latin-American Workshop on Nonlinear Phenomena in Fluids, Solids and Other Complex Systems (1991).

142. Chaos and complexity: The results of non-linear processes in the physical world, Evolutionary Trends in the Physical Sciences, Springer Proc. in Physics, 57, eds. M. Suzuki and R. Kubo (Springer-Verlag, 1991).

143. (with Peter Constantin) Dynamics of a complex interface, Physica D 47, 450-460 (1991).

144. (with W.-S. Dai and S.-M. Zhou) Interface dynamics and the motion of complex singularities, Phys. Rev. A43, 6672 (1991).

145. (with Michael J. Vinson and Amy J. Kolan) Chaos, computers, and physics, Laboratory Notes for Physics 251 (1987, 1990, 1991).

146. (with I. Procaccia, E. Ching, P. Constantin, A. Libchaber, and X-Z Wu) Transitions in convective turbulence: the role of thermal plumes, Phys. Rev. A44, 8091 (1991).

147. (with A. Libchaber, E. Moses, and G. Zocchi) Turbulence in a box, La Recherche 22, 628 (1991).

148. (with G. Vasconcelos) Stationary solutions for the Saffman-Taylor problem with surface tension, Phys. Rev. A44, 6490-6495 (1991).

149. Complex structures from simple systems, Phys. Today, 9 (March 1991).

150. Scaling and multiscaling: fracfals and multifractals (Review), Chinese J. Physics 29, 613-635 (1991).

151. (with Daniel H. Rothman) Bubble, bubble, boil, and trouble (in press).

152. Hard times, Phys. Today (October 1992)

153. (with Ashvin B. Chhabra, Amy J. Kolan, Mitchell J. Feigenbaum, and Itamar Procaccia) Critical indices for singular diffusion, Phys. Rev. A45, 6095 (1992).

154. (with Peter Constantin, Todd F. Dupont, Raymond E. Goldstein, Michael Shelley, and Su-Min Zhou) Droplet breakup in a model of the Hele Shaw cell, Phys. Rev. 
A (in press).

155. (with Ashvin B. Chhabra, Mitchell J. Feigenbaum, Amy J. Kolan, and Itamar Procaccia) Sandpiles, avalanches and the statistical mechanics of non-equilibrium stationary states, Phys. Rev. (in press). 